\title{
Rare decay searches at the Tevatron
}

\author{
C.-J. Lin ${ }^{\mathrm{a}}$ on behalf of the CDF and D $\varnothing$ Collaborations

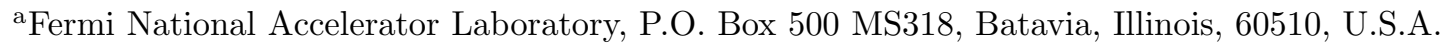

We present the current status of the rare $B$ decay searches $B_{s}^{0} \rightarrow \mu^{+} \mu^{-}, B^{0} \rightarrow \mu^{+} \mu^{-}, B^{+} \rightarrow K^{+} \mu^{+} \mu^{-}$, $B^{0} \rightarrow K^{* 0} \mu^{+} \mu^{-}$, and $B_{s}^{0} \rightarrow \phi \mu^{+} \mu^{-}$at the Tevatron.

\section{INTRODUCTION}

The flavor-changing-neutral-current (FCNC) transitions are highly suppressed in the Standard Model (SM) and can only occur through higher order diagrams. These processes can provide important tests of the SM at the level of radiative corrections where Glashow-Iliopoulos-Maiani (GIM) cancellations are important. Furthermore, in many new physics scenarios, additional loop diagrams involving new particles, such as Supersymmetric (SUSY) particles, can significantly alter the decay rates and kinematics of the FCNC processes. Therefore precision measurements of these rare processes are powerful probes of new physics and are complementary to direct collider searches.

At the Tevatron, we have searched for the FCNC decays of $B_{(s)}^{0} \rightarrow \mu^{+} \mu^{-}$. The SM expectations [1] for these branching fractions are significantly below the current experimental sensitivity. However, in many SUSY extensions of the SM, the branching fraction could be enhanced by one to three orders of magnitude to a level observable by the Tevatron experiments. An observation of these decays at the Tevatron would be unambiguous evidence for physics beyond the SM. In the absence of an observation, any improvements to the limits can be used to constrain significantly many SUSY models [2-4].

The $B \rightarrow X_{s} l^{+} l^{-}$transition is another important FCNC process that is accessible at the Tevatron. New physics effects could be seen in the forward-backward asymmetry of the strange meson in the dimuon system and/or in the decay rate [5]. At the Tevatron, we have searched for the following exclusive decay modes: $B^{+} \rightarrow$ $K^{+} \mu^{+} \mu^{-}, B^{0} \rightarrow K^{* 0} \mu^{+} \mu^{-}$, and $B_{s}^{0} \rightarrow \phi \mu^{+} \mu^{-}$. The former two decay modes have been observed at BaBar [6] and Belle [7]. The latter decay mode $B_{s}^{0} \rightarrow \phi \mu^{+} \mu^{-}$has so far evaded experimental detection.

\section{DETECTORS AND DATASETS}

The CDF [8] and DØ [9] detectors are generalpurpose particle detectors for studying $p \bar{p}$ collisions at a center-of-mass energy of $\sqrt{s}=1.96 \mathrm{TeV}$ at the Tevatron. Both detectors have good precision silicon vertex detectors and large muon coverage, which are important for the rare decay searches. Another crucial experimental component is the trigger system. At the Tevatron collision energy, the cross section for the inelastic QCD background is about 1000 times larger than the $b \bar{b}$ production. To suppress the large background, both experiments employ a sophisticated three-tier trigger system to filter collision events and select interesting physics events to store to tape for offline analysis. The data samples for the rare $B$ decay searches are primarily collected using the dimuon triggers. For the results presented here, the sample size used by the analyses ranges from $300 \mathrm{pb}^{-1}$ to $1 \mathrm{fb}^{-1}$.

\section{3. $B_{(s)}^{0} \rightarrow \mu^{+} \mu^{-}$SEARCHES}

The CDF and DØ experiments use similar strategy to search for the rare decay of $B_{s}^{0} \rightarrow$ $\mu^{+} \mu^{-}$. The offline analysis begins by selecting two muon candidates of opposite charge which satisfy the online dimuon trigger requirements. 
After applying some loose pre-selection requirements (e.g. transverse momentum $p_{T}$ of the muon and $B$ candidates), the selected sample is still dominated by combinatorial background and partially reconstructed $B$ hadrons.

For the final event selection, CDF uses the following four discriminating variables: dimuon invariant mass $M_{\mu \mu}$, proper decay length $\lambda=c \cdot M_{\mu \mu} L_{3 \mathrm{D}} /\left|\vec{p}^{\mu \mu}\right|$, the $3 \mathrm{D}$ opening angle $\Delta \Theta$ between vectors $\vec{p}^{\mu \mu}$ and $\vec{L}$, and $B$-candidate track isolation $I$. The vector $\vec{p}^{\mu \mu}$ is the momentum vector of the $B$ candidate, $L_{3 \mathrm{D}}$ is the $3 \mathrm{D}$ decay length projected on the $\vec{p}^{\mu \mu}$ direction, $\vec{L}$ is the displacement vector from the primary to the dimuon vertex, and the $B$-candidate track isolation is defined by $I=\left|\vec{p}_{T}^{\mu \mu}\right| /\left(\sum_{i} p_{T}^{i}+\right.$ $\left.\left|\vec{p}_{T}^{\mu \mu}\right|\right)$, where the sum is over all tracks with $\sqrt{\Delta \eta^{2}+\Delta \phi^{2}} \leq 1 ; \Delta \phi$ and $\Delta \eta$ are the azimuthal angle and pseudo-rapidity of track $i$ with respect to $\vec{p}^{\mu \mu}$. To enhance signal and background separation CDF constructs a multivariate likelihood ratio based on the latter three input variables: $I$, $\Delta \Theta$, and $\lambda$. We define the likelihood ratio to be

$L_{R}=\frac{\prod_{i} \mathbf{P}_{s}\left(x_{i}\right)}{\prod_{i} \mathbf{P}_{s}\left(x_{i}\right)+\prod_{i} \mathbf{P}_{b}\left(x_{i}\right)}$,

where $x_{1}=I, x_{2}=\Delta \Theta, x_{3}=\lambda$, and $\mathbf{P}_{s(b)}\left(x_{i}\right)$ is the probability that a signal (background) event has an observed $x_{i}$. The probability distributions for the signal events are obtained from the signal Monte Carlo (MC) and the background distributions are taken from the data sidebands. The resulting $L_{R}$ distributions for the signal and background events are shown in Fig. 1.

In the $\mathrm{D} \varnothing$ analysis background is suppressed by making stringent requirements on the variables $\Delta \Theta, I$, and the transverse decay length significance, $L_{2 \mathrm{D}} / \sigma\left(L_{2 \mathrm{D}}\right)$, where $L_{2 \mathrm{D}}$ is the projection of $\vec{L}$ onto the plane transverse to the beamline. The $L_{2 \mathrm{D}}$-significance is found to have a better discriminating power than $L_{2 \mathrm{D}}$ alone.

The $B_{s}^{0} \rightarrow \mu^{+} \mu^{-}$branching fraction is obtained by normalizing to the number of $B^{+} \rightarrow J / \psi K^{+} \rightarrow \mu^{+} \mu^{-} K^{+}$decays collected by the same trigger. With $780 \mathrm{pb}^{-1}\left(300 \mathrm{pb}^{-1}\right)$, CDF (DØ) reconstructs about $2400(900) B^{+}$ candidates. If no signal is observed, the upper limit on the branching fraction can be computed

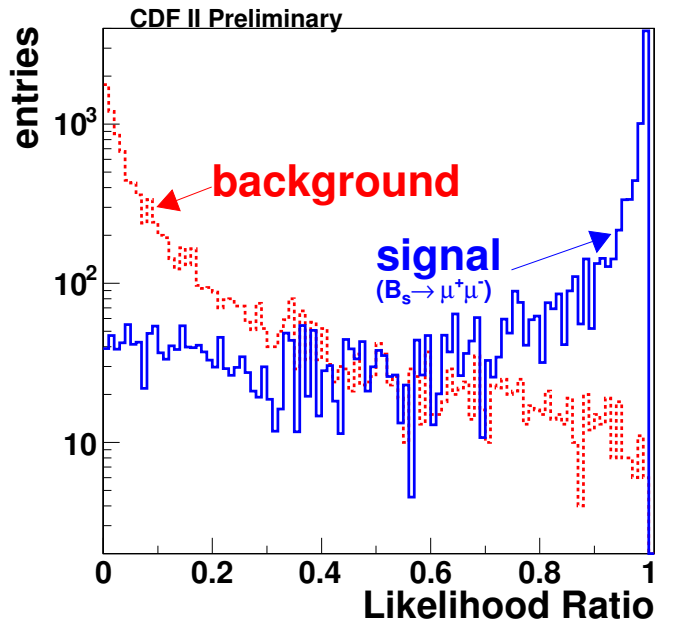

Figure 1. The likelihood ratio distribution for $B_{s}^{0} \rightarrow \mu^{+} \mu^{-}$signal (dashed) from PYTHIA MC and background (solid) from data sidebands.

using the expression

$$
\begin{gathered}
\mathcal{B}\left(B_{s}^{0} \rightarrow \mu^{+} \mu^{-}\right)^{90 \% C \cdot L .}=\frac{N_{B_{s}^{0}}^{90 \%}}{N_{B^{+}}} \cdot \frac{\epsilon_{B^{+}}}{\epsilon_{B_{s}^{0}}} . \\
\frac{f_{u}}{f_{s}} \cdot \mathcal{B}\left(B^{+} \rightarrow J / \psi K^{+} \rightarrow \mu^{+} \mu^{-} K^{+}\right),
\end{gathered}
$$

where $N_{B_{s}^{0}}^{90 \%}$ is the number of $B_{s}^{0} \rightarrow \mu^{+} \mu^{-}$decays at the $90 \%$ C.L. for $N$ observed and $N_{B}$ expected background events. The value of $N_{B_{s}^{0}}^{90 \%}$ is estimated using the Bayesian approach assuming a flat prior for the $\mathcal{B}\left(B_{s}^{0} \rightarrow \mu^{+} \mu^{-}\right)$and incorporating Gaussian uncertainties into the limit. The parameter $\epsilon_{B_{s}^{0}}\left(\epsilon_{B^{+}}\right)$is the total efficiency of the for triggering and reconstructing the signal (normalization) mode. We use the world average fragmentation ratio $f_{u} / f_{s}$ and branching fraction $\mathcal{B}\left(B^{+} \rightarrow J / \psi K^{+} \rightarrow \mu^{+} \mu^{-} K^{+}\right)$.

CDF optimizes the analysis based on the $a$ priori expected $90 \%$ C.L. upper limit on $\mathcal{B}\left(B_{s, d}^{0} \rightarrow\right.$ $\left.\mu^{+} \mu^{-}\right)$. The resulting optimal criteria is $L_{R}>$ 0.99 . With the optimized selection requirements and $780 \mathrm{pb}^{-1}$, there are 1 (2) candidates in the $B_{s}^{0} \rightarrow \mu^{+} \mu^{-}\left(B^{0} \rightarrow \mu^{+} \mu^{-}\right)$signal window, as shown in Fig. 2, with an expected background of $1.3 \pm 0.4(2.5 \pm 0.4)$. CDF defines the signal windows as $\pm 70 \mathrm{MeV}$ about the world aver- 
age $B_{s}^{0}\left(B^{0}\right)$ mass, which corresponds to about \pm 2.5 times the mass resolution. The expected background is estimated by extrpolating events from the sidebands to the signal region. Given the number of signal events observed is consistent with the background expectation, we computed the following $90 \%$ (95\%) C.L. limits of $\mathcal{B}\left(B_{s}^{0} \rightarrow \mu^{+} \mu^{-}\right)<8.0 \times 10^{-8}\left(1.0 \times 10^{-7}\right)$ and $\mathcal{B}\left(B^{0} \rightarrow \mu^{+} \mu^{-}\right)<2.3 \times 10^{-8}\left(3.0 \times 10^{-8}\right)$.

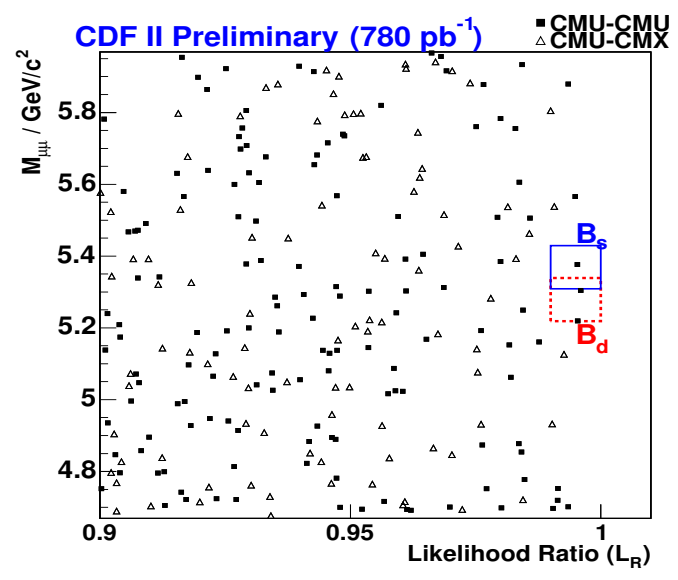

Figure 2. The $\mu^{+} \mu^{-}$invariant mass versus likelihood ratio for central-central (CMU-CMU) and central-forward (CMU-CMX) dimuon trigger samples. The $B_{s}^{0}$ (solid box) and $B^{0}$ (dashed box) signal regions are also shown.

$\mathrm{D} \varnothing$ optimizes the three discriminating variables using a random-grid search. The optimization resulted in this choice of final selection criteria: $\Delta \Theta<0.2 \mathrm{rad}, I>0.56$, and $L_{2 \mathrm{D}} / \sigma\left(L_{2 \mathrm{D}}\right)>18.5$. Based on $300 \mathrm{pb}^{-1}$ of data, there are 4 candidate events in the signal window of $\pm 180 \mathrm{MeVabout}$ the world average $B_{s}^{0}$ mass, as shown in Fig. 3, with an expected background of 4.3 \pm 1.2 . The $\mathrm{D} \varnothing$ mass resolution is insufficient to discriminate $B_{s}^{0}$ from $B^{0} \rightarrow \mu^{+} \mu^{-}$decays. The branching fraction limit is computed assuming the contribution from $B^{0}$ is negligible, which is a good approximation for minimal-flavor-violating models. The resulting limit at $90 \%(95 \%)$ C.L. is $\mathcal{B}\left(B_{s}^{0} \rightarrow \mu^{+} \mu^{-}\right)<3.2 \times 10^{-7}\left(4.0 \times 10^{-7}\right)$. Using the same analysis technique, $\mathrm{D} \varnothing$ estimated the expected $95 \%$ C.L. limit with $700 \mathrm{pb}^{-1}$ to be $\mathcal{B}\left(B_{s}^{0} \rightarrow \mu^{+} \mu^{-}\right)<2.3 \times 10^{-7}$.

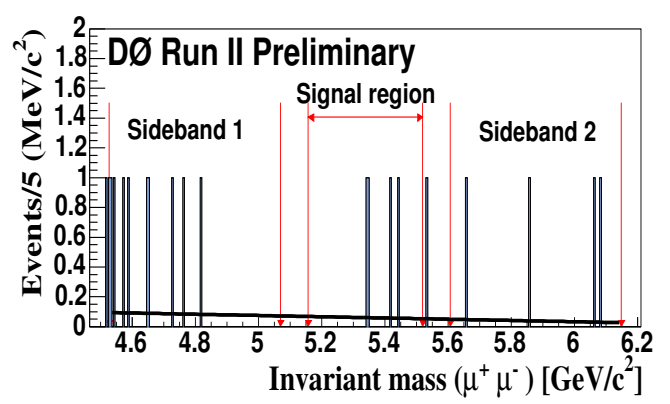

Figure 3. The dimuon mass distribution based on $300 \mathrm{pb}^{-1}$ of D $\varnothing$ data. Four events are observed in the signal region.

\section{EXCLUSIVE $B \rightarrow K^{(*)} \mu^{+} \mu^{-}$SEARCHES}

In additional to the two-body dimuon decays, the Tevatron has also searched for the $b \rightarrow$ $s \mu^{+} \mu^{-}$FCNC processes. In particular, CDF has searched for the decays of $B^{+} \rightarrow K^{+} \mu^{+} \mu^{-}, B^{0} \rightarrow$ $K^{* 0} \mu^{+} \mu^{-}$, and $B_{s}^{0} \rightarrow \phi \mu^{+} \mu^{-}$in $1 \mathrm{fb}^{-1}$ of data. $\mathrm{D} \varnothing$ has focused the search in the $B_{s}^{0} \rightarrow \phi \mu^{+} \mu^{-}$ channel using $450 \mathrm{pb}^{-1}$ of data.

The offline analysis begins by searching for a pair of oppositely charged muon tracks. The two muon tracks are combined with a third charged track to from a $B^{+} \rightarrow K^{+} \mu^{+} \mu^{-}$candidate, or another pair of oppositely charged tracks to from a $B^{0} \rightarrow K^{* 0} \mu^{+} \mu^{-}$or $B_{s}^{0} \rightarrow \phi \mu^{+} \mu^{-}$candidate. The $K^{* 0}$ is reconstructed in the mode $K^{* 0} \rightarrow K^{+} \pi^{-}$and the $\phi$ is reconstructed as $\phi \rightarrow K^{+} K^{-}$. We exclude events where the dimuon invariant mass is within the $J / \psi \rightarrow \mu^{+} \mu^{-}$ and $\psi(2 S) \rightarrow \mu^{+} \mu^{-}$mass regions to eliminate possible contributions from the charmonium resonant decays.

In the CDF search, muons are required to have $p_{T}>1.5$ or $2.0 \mathrm{GeV}$ depending on which dimuon trigger selected the event. The kaon requirement is $p_{T}>0.4 \mathrm{GeV}$. CDF uses the following three discriminating variables in the optimization of the searches. The first variable is the proper decay length significance $\lambda_{2 D} / \sigma\left(\lambda_{2 D}\right)$. The $\lambda_{2 D}$ vari- 

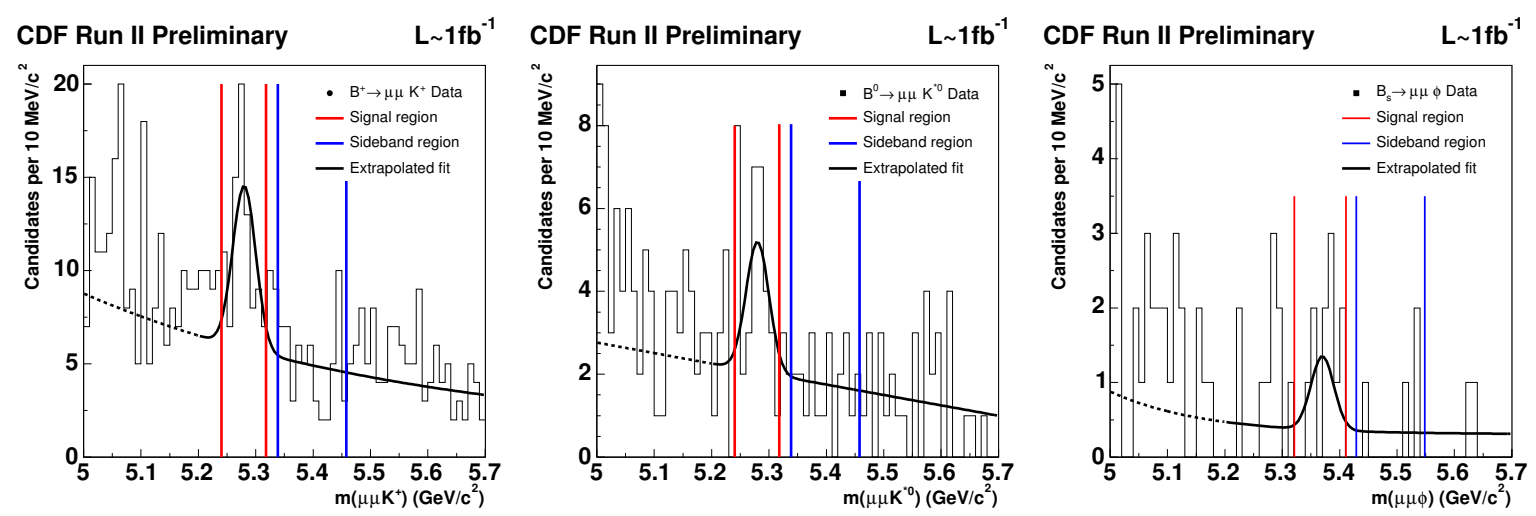

Figure 4. The invariant distribution for the three rare decay modes. The vertical bars define the signal and sideband regions. The black curve illustrates the expected shape for the signal and combinatoric background.

able is defined similarly to the $\lambda$ variable described earlier, with $L_{3 \mathrm{D}}$ replaced by the $2 \mathrm{D}$ decay length $L_{2 \mathrm{D}}$. The second variable is the $2 \mathrm{D}$ opening angle $\Delta \alpha$, which is angle between the momentum vector of the $B$ candidate and the decay axis $\vec{L}$ projected onto the transverse plane. The final discriminating variable is the $B$ candidate isolation $I$. The expected number of background events in the $B$ mass window is obtained by extrapolating events in the high-mass sideband to the signal region. Since the region below the $B$ signal window contains partially reconstructed $B$ decays, only the high-mass sideband is used in the background estimate. The figure-of-merit for the optimization is $S / \sqrt{(} S+B)$, where $S$ is the estimate of the expected yield of the rare decays, and $B$ is the expected background. For the $B^{+}$ and $B^{0}$ rare decay searches, the PDG values of the branching fractions are used in the optimization, while the theoretical expectation is used for the $B_{s}^{0}$ search. The optimization is performed separately for the three rare decay modes. The resulting optimal values are very similar between the different modes and the following averages are used for all three searches: $\lambda_{2 D} / \sigma\left(\lambda_{2 D}\right)>14$, $\Delta \alpha<0.06 \mathrm{rad}$, and $I>0.6$. The invariant mass distribution for the three searches after applying the optimal requirements on the discriminating variables are shown in Fig. 4.

In the $\mathrm{D} \varnothing$ search, muons are required to have $p_{T}>2.5 \mathrm{GeV}$ and kaon with $p_{T}>0.4 \mathrm{GeV}$. The $\mathrm{D} \varnothing$ search for the $B_{s}^{0} \rightarrow \phi \mu^{+} \mu^{-}$decay is op- timized using the following three discriminating variables: 2D pointing angle $\Delta \alpha, B$ candidate isolation $I$, and the $2 \mathrm{D}$ decay length significance $L_{2 \mathrm{D}} / \sigma\left(L_{2 \mathrm{D}}\right)$. D $\varnothing$ uses a random-grid search to find the optimal values of the discriminants by maximizing the figure-of-merit $\epsilon_{S} /(a / 2+\sqrt{B}$. The parameter $\epsilon_{S}$ is the efficiency for reconstructing the signal and the constant $a$ is set to 2.0, corresponding to testing the signal hypothesis at the $95 \%$ C.L. The number of background events is estimated by extrapolating the high and lowmass sidebands to the signal window. The optimization procedure yields the following values for the discriminating variables: $\Delta \alpha<0.1 \mathrm{rad}$, $I>0.72$, and $L_{2 \mathrm{D}} / \sigma\left(L_{2 \mathrm{D}}\right)>10.3$. The invariant mass distribution after applying optimized selection requirements is shown in Fig. 5 .

The branching fraction can be computed by normalizing the number of the observed signal to the number of reconstructed resonant $B \rightarrow h J / \psi$ decays:

$$
\begin{aligned}
\frac{\mathcal{B}\left(B \rightarrow h \mu^{+} \mu^{-}\right)}{\mathcal{B}(B \rightarrow h J / \psi)}=\frac{N_{h \mu^{+}+\mu^{-}}}{N_{h J / \psi}} \cdot \frac{\epsilon_{h J / \psi}}{\epsilon_{h \mu^{+} \mu^{-}}} . \\
\mathcal{B}\left(J / \psi \rightarrow \mu^{+} \mu^{-}\right),
\end{aligned}
$$

where $h$ is $K^{+}, K^{* 0}$, or $\phi$. The parameter $N_{h \mu^{+} \mu^{-}}$is either the number of observed signal events or in the case of setting a limit, the upper limit on the number of signal decays, and $N_{h J / \psi}$ is the number of reconstructed $B \rightarrow h J / \psi$ events. The efficiency terms $\epsilon_{h J / \psi}$ and $\epsilon_{h \mu^{+} \mu^{-}}$are the efficiency for reconstructing the normalization and 


\begin{tabular}{|l|c|c|c|c|}
\hline $\begin{array}{l}\text { Experiment } \\
\text { Mode }\end{array}$ & $\begin{array}{c}\text { CDF } \\
B^{+} \rightarrow \mu^{+} \mu^{-} K^{+}\end{array}$ & $\begin{array}{c}\text { CDF } \\
B_{d}^{0} \rightarrow \mu^{+} \mu^{-} K^{* 0}\end{array}$ & $\begin{array}{c}\text { CDF } \\
B_{s}^{0} \rightarrow \mu^{+} \mu^{-} \phi\end{array}$ & $\begin{array}{c}\text { D } \varnothing \\
B_{s}^{0} \rightarrow \mu^{+} \mu^{-} \phi\end{array}$ \\
\hline Signal Yield & 90 & 35 & 11 & 0 \\
\hline Expected BKG & $45.3 \pm 5.8$ & $16.5 \pm 3.6$ & $3.5 \pm 1.5$ & $1.6 \pm 0.4$ \\
\hline Gaussian significance & 4.5 & 2.9 & 2.4 & - \\
\hline Rel $\mathcal{B} \times 10^{-3}$ & $0.59 \pm 0.15 \pm 0.03$ & $0.62 \pm 0.23 \pm 0.07$ & $1.24 \pm 0.60 \pm 0.15$ & - \\
\hline Abs $\mathcal{B} \times 10^{-6}$ & $0.60 \pm 0.15 \pm 0.04$ & $0.82 \pm 0.31 \pm 0.10$ & $1.16 \pm 0.56 \pm 0.42$ & - \\
\hline $\operatorname{Rel} \mathcal{B} 95 \%$ CL limit $\times 10^{-3}$ & - & - & 2.61 & 7.4 \\
\hline $\operatorname{Rel} \mathcal{B} 90 \%$ CL limit $\times 10^{-3}$ & - & - & 2.30 & 5.6 \\
\hline
\end{tabular}

Table 1

Summary of the $B \rightarrow h \mu^{+} \mu^{-}$searches at CDF and DØ. The branching fraction limits are computed using Bayesian method.

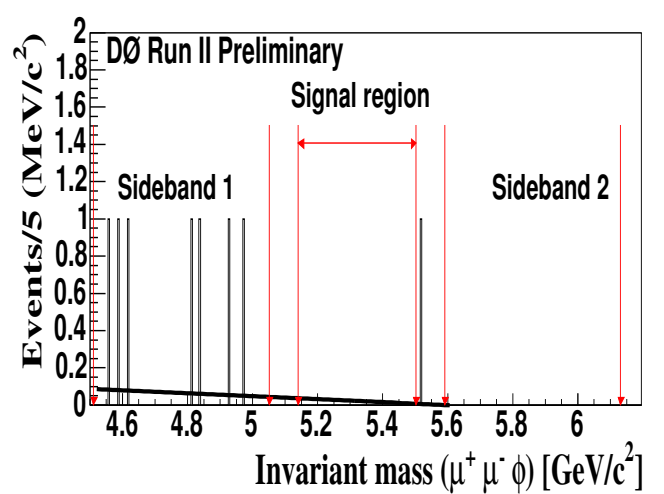

Figure 5. The invariant distribution for the $B_{s}^{0} \rightarrow$ $\phi \mu^{+} \mu^{-}$mode. The solid line shows the sidebands background interpolation into the signal region.

signal decays, respectively.

The branching fraction results and limits are tabulated in Tab. 1. CDF has seen strong evidence of the rare decays in the $B^{+}$and $B^{0}$ channels. Neither CDF nor DØ observed significant signal in the $B_{s}^{0}$ channel.

\section{CONCLUSIONS}

With a subset of currently available dataset, $\mathrm{CDF}$ and $\mathrm{D} \varnothing$ have made important progress in rare decay searches. The current limit of $B_{s}^{0} \rightarrow$ $\mu^{+} \mu^{-}$is already significantly constraining some scenarios of new physics beyond the SM In addition, CDF has seen significant evidence of signal in the $B^{+} \rightarrow K^{+} \mu^{+} \mu^{-}$and $B^{0} \rightarrow K^{* 0} \mu^{+} \mu^{-}$ modes. So far, no signal is observed in the $B_{s}^{0} \rightarrow \phi \mu^{+} \mu^{-}$channel. However, the Tevatron is reaching the SM level of sensitivity; an observation of this mode is expected in the near term.

\section{REFERENCES}

1. G. Buchalla and A. J. Buras, Nucl. Phys. B400, 225 (1993); A.J. Buras, Phys. Lett. B 566, 115 (2003).

2. S. Choudhury and N. Gaur, Phys. Lett. B 451, 86 (1999); K.S. Babu and C. Kolda, Phys. Rev. Lett. 84, 228 (2000).

3. R. Dermisek et al., J. High Energy Phys. 04, 037 (2003); D. Auto et al., J. High Energy Phys. 06, 023 (2003).

4. H. Baer et al., J. High Energy Phys. 07, 050 (2002); S. Baek, P. Ko, and W.Y. Song, Phys. Rev. Lett. 89, 271801 (2002); H. Logan and U. Nierste, Phys. Lett. B 566, 115 (2003); A. Dedes and B. Huffman, Phys. Lett. B 600 , 261 (2004).

5. P. Colangelo, F. De Fazio, R. Ferrandes and T. N. Pham, Phys. Rev. D 73, 115006 (2006); F. Kruger and J. Matias, Phys. Rev. D 71, 094009 (2005); T. M. Aliev, A. Ozpineci and M. Savci, Eur. Phys. J. C 29, 265 (2003).

6. B. Aubert et al., BABAR Collaboration, Phys. Rev. D 73, 092001 (2006).

7. A. Ishikawa et al., BELLE Collaboration, Phys. Rev. Lett. 96, 251801 (2006).

8. D. Acosta et al., CDF Collaboration, Phys. Rev. D 71, 032001 (2005).

9. V. .M. Abazov et al., D $\varnothing$ Collaboration, arXiv:physics/0507191. 